\title{
Novel Synthesis of 1,2-Substituted 4-Quinolones
}

\author{
Sreenivasulareddy Bandatmakuru \\ Veerareddy Arava* (1)
}

R\&D Centre, Suven Life Sciences Ltd, Plot No\#18, Phase-III, IDA, Jeedimetla, Hyderabad-500055, India

reddyvenis@rediffmail.com

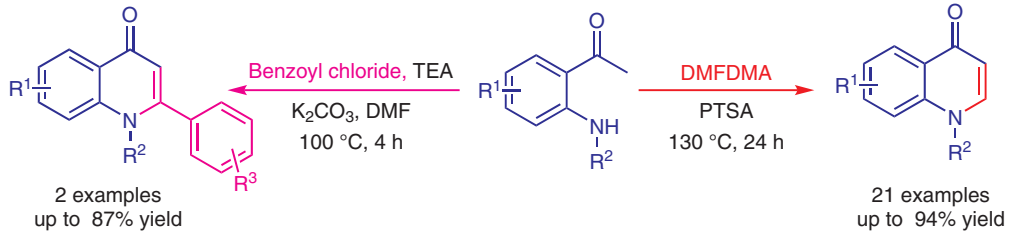

Received: 21.08.2018

Accepted after revision: 25.10 .2018

Published online: 21.11 .2018

DOI: 10.1055/s-0037-1610388; Art ID: so-2018-d0049-op

License terms: CC $\cong \$$

Abstract An efficient method for the straightforward synthesis of $\mathrm{N}$ functionalized 4-quinolones and 1,2-substituted 4-quinolones from simple 2-aminoacetophenones has been developed.

Key words 2-aminoacetophenone, 4-quinolone, echinopsine

Nitrogen-containing heterocycles are frequently found in a variety of biologically active molecules that can be used in therapeutic areas. ${ }^{1}$ Specifically, 4 -quinolone derivatives have attracted considerable attention because of their diverse biological activities. Several quinolone compounds, such as oxolinic acid, Ciprofloxacin, Pefloxacin, and Ofloxacin, have emerged as potent antibiotics (Figure 1). ${ }^{2}$<smiles>CCn1cc(C(=O)O)c(=O)c2cc3c(cc21)OCO3</smiles><smiles>O=C(O)c1cn(C2CC2)c2cc(N3CCNCC3)c(OC3CCCC3)cc2c1=O</smiles><smiles>CCn1cc(C(=O)O)c(=O)c2cc(F)c(N3CCN(C)CC3)cc21</smiles><smiles>CC1COc2c(N3CCN(C)CC3)c(F)cc3c(=O)c(C(=O)O)cn1c23</smiles>

Figure 1 Representative potent antibiotics containing the 4-quinolone moiety

More recently, 4-quinolone derivatives have been explored for their antibacterial, ${ }^{3}$ antitumor, ${ }^{4}$ antimalarial, ${ }^{5}$ antidiabetic, ${ }^{6}$ antiviral $^{7}$ and HIV-1 integrase inhibition proper- ties. ${ }^{8}$ Given the importance of these heterocycles in medical chemistry, the development of synthetic methodology to access 4-quinolone derivatives remains an imperative. To date, numerous methods have been reported for the synthesis of quinolones. ${ }^{9}$ The most frequently used approaches are based on various cyclocondensation strategies, such as the Camps, ${ }^{10}$ Conrad-Limpach, ${ }^{11}$ Gould-Jacobs, ${ }^{12}$ and Niementowski cyclizations. ${ }^{13}$ Often these synthetic methods are carried out under extremely harsh conditions, including temperatures up to $250{ }^{\circ} \mathrm{C}$ or the use of strong acids such as polyphosphoric acid or Eaton's reagent. As a result, the harsh conditions dramatically limit the substrate scope of these transformations. To develop milder processes for construction of the 4-quinolone framework, much effort has been focused on the development of transition-metal-catalyzed $\left(\mathrm{Pd},{ }^{14} \mathrm{Cu},{ }^{15}\right.$ and $\left.\mathrm{Au}^{16}\right)$ cyclization methodologies during the past decade. Despite significant progress, transition-metal-catalyzed synthetic methods often require specially designed ligands. Another disadvantage is the need to remove metal-related impurities from products, which is an important issue in the synthesis of pharmaceutical molecules.

Some quinolones have been found to be active as mammalian topoisomerase-II inhibitors, including a series of 3unsubstituted compounds. ${ }^{17}$ 1-Methyl-1,4-dihydroquinolin-4-one, echinopsine, ${ }^{18}$ is a nontoxic alkaloid from Echinops species that regulates the function of the parasympathetic autonomous nervous system. ${ }^{19}$ Several 1 -alkyl-3-unsubstituted derivatives have been prepared by decarboxylation of the corresponding 3-carboxylic acids. ${ }^{20}$ This method usually requires high temperatures and the reported yields are generally low to medium. Some 1-aryl-3unsubstituted 1,4-dihydroquinolin-4-ones have also been prepared by this method but the yields are also generally low. ${ }^{17}$ Thermal rearrangement of 4-methoxy- and 4ethoxyquinoline derivatives can be used for the synthesis of the corresponding 1-methyl- and 1-ethyl-1,4-dihydroquin- 
olin-4-ones, respectively. ${ }^{21}$ This method usually requires high temperatures $\left(300-350{ }^{\circ} \mathrm{C}\right.$ ) and the yields are again usually low. Lower temperatures and higher yields were reported when the rearrangement was carried out in the presence of the appropriate iodoalkane, ${ }^{22}$ alkyl tosylate, ${ }^{23}$ or trialkyl phosphate. ${ }^{23}$ 1-Alkyl-3-unsubstituted 1,4-dihydroquinolin-4-ones having a primary alkyl group at the 1-position can also be prepared by $\mathrm{N}$-alkylation of the corresponding 1-unsubstituted 1,4-dihydroquinolin-4-ones.

It is known that amino-substituted acetophenones are valuable precursors for the synthesis of medicinally important substances such as 2-arylquinolin-4(1H)-ones and their analogues. ${ }^{24,25}$ In recent years, interest in these compounds has prompted extensive studies into their properties, such as toxicity to human tumor cell lines and tubulin polymerization inhibition. ${ }^{4 a, 26}$ The method most widely used to prepare 2-aryl-2,3-dihydroquinolin-4(1H)-ones includes a two-step sequence consisting of base-catalyzed aldol condensation of 2-aminoacetophenones and aldehydes and then acid-catalyzed cyclization of the corresponding 2-aminochalcones thus formed via an intramolecular aza-Michael reaction. ${ }^{25-27}$ Other groups have also investigated the synthesis of 4-quinolones from 2-aminoacetophenones, ${ }^{28}$ 2-bromoacetophenones, ${ }^{14 \mathrm{~d}}$ 2-halophenones, ${ }^{15 \mathrm{a}}$ and 2-iodoanilines, ${ }^{29}$ as well as the reactions of isatoic anhydrides with aryl ketones ${ }^{30 \mathrm{a}}$ or alkynes ${ }^{30 \mathrm{~b}}$ using transition-metal catalysts. Tambe and co-workers used copper-mediated Ncyclopropylation on substituted fused or unfused pyridinol systems to generate $N$-cyclopropyl quinolones in moderate yields (Equation 1). ${ }^{31}$
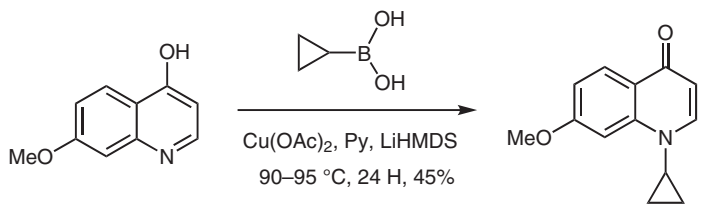

Equation 1

Kumar et al. synthesized $N$-aryl quinolones from quinolone and diaryliodonium salts in good yields (Equation 2). ${ }^{32}$

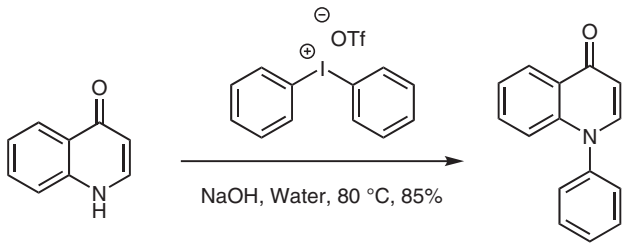

Equation 2

Ueno et al. prepared $N$-alkyl quinolones by the nickelcatalyzed intramolecular amination of 2-( $\mathrm{N}$-alkylamino)propiophenones at the $\beta$-carbon in good yields (Equation 3$){ }^{18 \mathrm{j}}$

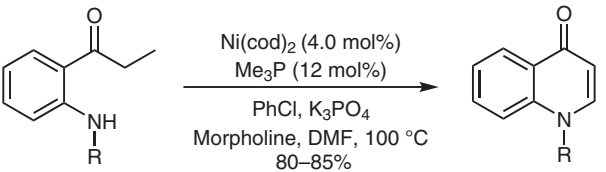

Equation 3

Shao et al.9h prepared $N$-cyclopropyl quinolones from trimethylsilyl substituted substrates and cyclopropyl amine in good yields (Equation 4).

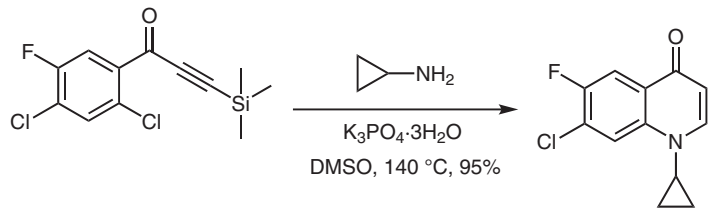

Equation 4

However, especially for structure-activity studies, the need for new methods for the preparation of the 3-unsubstituted compounds remains. This is particularly true for 1sec-alkyl, 1-tert-alkyl, and 1-aryl-1,4-dihydroquinolin-4ones.

At the outset, when we attempted the reaction of 1-(2cyclopropylaminophenyl)ethanone ${ }^{33}$ with dimethylformamide dimethylacetal (DMFDMA) as both reactant and solvent, the desired product 2a was not observed (Table 1). However, product 2a was formed in 90\% yield when paratoluenesulfonic acid (PTSA, $0.1 \mathrm{~mol}$ ) in ortho-xylene was employed (entry 10). The yields were not improved by using other acids such as methanesulfonic acid, benzenesulfonic acid, camphor sulfonic acid, conc. $\mathrm{HCl}$ or sulfuric acid (entries 11-15). A survey of reaction media showed that the use of polar solvents such as DMSO, DMF, and DMA provided better results than those obtained in either toluene or 1,4-dioxane (entries 16-21).

A series of experiments were then carried out to reveal the crucial role of the reaction temperature (Table 1, entries 4-9). The results showed that increasing reaction temperature led to higher yields ( $90 \%$ at $130{ }^{\circ} \mathrm{C}$ vs. $25 \%$ at $100{ }^{\circ} \mathrm{C}$; entries 10 and 5). Investigation of the effect of time on the reaction showed that higher yields can be obtained by prolonging the reaction time from 8 to 24 hours (entries 8-10). Thus, optimal conditions used 1a and DMFDMA in the presence of PTSA in ortho-xylene at $130{ }^{\circ} \mathrm{C}$ (entry 10).

With the optimized reaction conditions established, we then studied the scope of the cyclization of DMFDMA with a series of other aminoacetophenones, as shown in Scheme 1. First, we examined the effect of substitution with electron-donating groups and electron-withdrawing groups (EWGS) on the phenyl ring. Both were well tolerated and gave the corresponding quinolones in good to excellent yields (60-90\%). All ortho-, meta- and para-substituted 
Table 1 Optimization of One-Pot Tandem Reaction Conditions of 2a

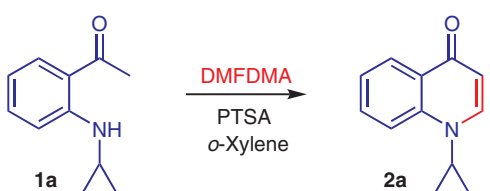

\begin{tabular}{llllll}
\hline Entry & Solvent & Catalyst & $\begin{array}{l}\text { Temp } \\
\left({ }^{\circ} \mathrm{C}\right)\end{array}$ & $\begin{array}{l}\text { Time } \\
(\mathrm{h})\end{array}$ & Yield (\%) \\
\hline 1 & DMFDMA & - & 80 & 24 & $\mathrm{NR}$ \\
2 & DMFDMA & - & 100 & 24 & $\mathrm{NR}$ \\
3 & DMFDMA & - & 130 & 24 & $\mathrm{NR}$ \\
4 & ortho-xylene & PTSA & 80 & 40 & 10 \\
5 & ortho-xylene & PTSA & 100 & 38 & 25 \\
6 & ortho-xylene & PTSA & 110 & 38 & 33 \\
7 & ortho-xylene & PTSA & 120 & 38 & 42 \\
8 & ortho-xylene & PTSA & 130 & 12 & 63 \\
9 & ortho-xylene & PTSA & 130 & 20 & 82 \\
10 & ortho-xylene & PTSA & 130 & 24 & 90 \\
11 & ortho-xylene & methanesulfonic acid & 130 & 24 & 10 \\
12 & ortho-xylene & benzenesulfonic acid & 130 & 24 & 15 \\
13 & ortho-xylene & camphorsulfonic acid & 130 & 24 & 15 \\
14 & ortho-xylene & Conc. HCl & 130 & 24 & 50 \\
15 & ortho-xylene & Conc. $\mathrm{H}_{2}$ SO $\mathrm{H}_{4}$ & 130 & 24 & NR \\
16 & DMF & PTSA & 130 & 24 & 65 \\
17 & DMSO & PTSA & 130 & 24 & 65 \\
18 & chlorobenzene & PTSA & 130 & 24 & 55 \\
19 & toluene & PTSA & 110 & 24 & trace \\
20 & dioxane & PTSA & 100 & 24 & NR \\
21 & DMA & PTSA & 130 & 24 & trace \\
\hline & & & &
\end{tabular}

aminoacetophenones were smoothly transformed into the desired products, which indicates that steric bulk and electronic effects did not significantly alter the reactivity.

To explore substrate scope still further, we next examined variations in the nitrogen substituent $R^{2}$. When $R^{2}$ was cyclic (cyclopentyl, cyclohexyl), all substrates examined were smoothly converted into the corresponding quinolones $\mathbf{2 h}-\mathbf{k}$ (Scheme 2). The method was successfully utilized in the synthesis of echinopsine 2 l. Changing $\mathrm{R}^{2}$ to an aryl group led to quinolones $\mathbf{2 m}-\mathbf{u}$ in good yields. Substrates possessing $\mathrm{N}$-aryl substituents containing either electrondonating or electron-withdrawing groups also reacted efficiently.

We also evaluated the possibility of synthesizing 1,2disubstituted 4-quinolones 4a directly from 2-aminoacetophenone 1a and benzoyl chloride, using TEA as the cata-
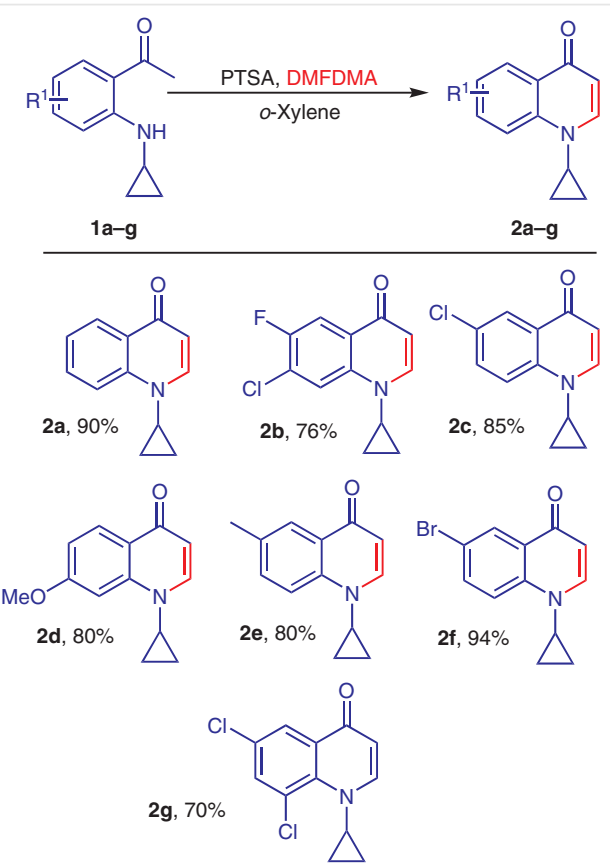

Scheme 1 One-pot synthesis of N-substituted-4-quinolone derivatives

lyst and THF as the solvent. Subsequently, the intermediate was cyclized with DMF and $\mathrm{K}_{2} \mathrm{CO}_{3}$ and the desired product 4a was formed in $87 \%$ yield (Scheme 3 ).

Quinolones 4 were useful synthetic precursors; for example, the corresponding 3-functionalized quinolones can be readily generated by using well-documented amination, ${ }^{34}$ cyanation, ${ }^{35}$ Heck, ${ }^{5 a, 36}$ Sonogashira, ${ }^{37}$ and SuzukiMiyaura $^{14,38}$ reactions from 3-halogenated quinolones prepared by direct halogenation of products $4 .{ }^{14 e, 38 b}$

In summary, we have developed an efficient method for the straightforward synthesis of $\mathrm{N}$-functionalized 4-quinolones and 1,2-substituted 4-quinolones from 2-aminoacetophenones. By using this method, $\mathrm{N}$-alkyl and $\mathrm{N}$-aryl aminoacetophenones can be successfully transformed into the corresponding 4-quinolones. This approach provides one of the simplest methods for the synthesis of this class of compounds, and a wide range of multisubstituted 4-quinolones can be generated accordingly.

\section{Preparation of 1-Cyclopropyl-1H-quinolin-4-one (2a); Typical Pro-} cedure

A mixture of 1-(2-cyclopropylamino-phenyl)ethanone 1 (1.0 gm, 5.71 $\mathrm{mmol})$, dimethylformamide dimethylacetal $(2.0 \mathrm{~mL})$, and PTSA (100 $\mathrm{mg}, 0.571 \mathrm{mmol})$ in 0 -xylene $(30 \mathrm{~mL})$ was heated to reflux for $24 \mathrm{~h}$. After completion of reaction (monitored by TLC), the reaction mixture was allowed to cool and then diluted with $o$-xylene $(10 \mathrm{~mL})$. Water $(20 \mathrm{~mL})$ was added and the organic phase was separated. The aqueous layer was then extracted further with $o$-xylene $(10 \mathrm{~mL})$ and the combined organic extracts were washed with brine, dried over sodium sulfate, filtered and concentrated under reduced pressure to give $\mathbf{2 a}$. 
<smiles>CCc1cc[nH+]cc1CC</smiles><smiles>CC(=O)c1cc[nH+]cc1NP</smiles><smiles></smiles><smiles>O=c1ccn(C2CCCCC2)c2ccccc12</smiles><smiles>O=c1ccn(C2CCCC2)c2ccc(Cl)cc12</smiles>

2h, $84 \%$<smiles>Cn1ccc(=O)c2ccccc21</smiles><smiles>O=c1ccn(-c2ccc(Cl)cc2)c2ccccc12</smiles><smiles>Cc1ccc2c(c1)c(=O)ccn2-c1ccc(Br)cc1</smiles><smiles>O=c1ccn(-c2ccc(Br)cc2)c2ccccc12</smiles>
2n, $88 \%$<smiles>Cc1ccc2c(c1)c(=O)ccn2-c1ccccc1</smiles><smiles>O=c1ccn(-c2ccccc2)c2ccc(Br)cc12</smiles>

2o, $84 \%$<smiles>O=c1ccn(-c2ccccc2)c2ccc(Cl)cc12</smiles>

$2 p, 86 \%$
2 r, $80 \%$

2s, $84 \%$<smiles>O=c1ccn(-c2ccc([N+](=O)[O-])cc2)c2ccccc12</smiles>

2t, $85 \%$<smiles>COc1cccc(-n2ccc(=O)c3ccccc32)c1</smiles>

$2 u, 82 \%$

Scheme 2 Synthesis of $\mathrm{N}$-substituted-4-quinolone derivatives

Yield: $950 \mathrm{mg}$ (90\%); yellow solid; $\mathrm{mp} 79.8-81.4{ }^{\circ} \mathrm{C}$. IR (KBr): 3488, 1610, 1620, 1565, 1485, 1299, $762 \mathrm{~cm}^{-1}$.

${ }^{1} \mathrm{H}$ NMR (400 MHz, $\mathrm{CDCl}_{3}$ ): $\delta=1.03-1.12(\mathrm{q}, 2 \mathrm{H}), 1.23-1.30(\mathrm{q}, 2 \mathrm{H})$, 3.36-3.42 (m, $1 \mathrm{H}), 6.23(\mathrm{~d}, J=7.92 \mathrm{~Hz}, 1 \mathrm{H}), 7.37-7.41(\mathrm{~m}, 1 \mathrm{H}), 7.66-$ $7.70(\mathrm{~m}, 2 \mathrm{H}), 7.91(\mathrm{~d}, J=8.6 \mathrm{~Hz}, 1 \mathrm{H}), 8.42\left(\mathrm{dd}, J_{1}=0.84, J_{2}=0.88 \mathrm{~Hz}\right.$, $1 \mathrm{H})$.

${ }^{13} \mathrm{C}$ NMR $\left(100 \mathrm{MHz}, \mathrm{CDCl}_{3}\right): \delta=8.19,33.61,109.89,116.91,123.75$, $126.67,131.96,141.51,142.51,178.33$.

Anal. Calcd for $\mathrm{C}_{12} \mathrm{H}_{11} \mathrm{NO}$ : C, 77.81; H, 5.99; N, 7.56; Found: C, 77.80; H, 5.97; N, 7.53 .

\section{7-Chloro-1-cyclopropyl-6-fluoro-1H-quinolin-4-one (2b)}

Yield: $396 \mathrm{mg}$ (76\%); yellow solid; $\mathrm{mp} 195.6-197.2{ }^{\circ} \mathrm{C}$.

IR (KBr): 3100, 3027, 1633, 1610, 1589, 1477, 1259, 971, 893, $824 \mathrm{~cm}^{-1}$.

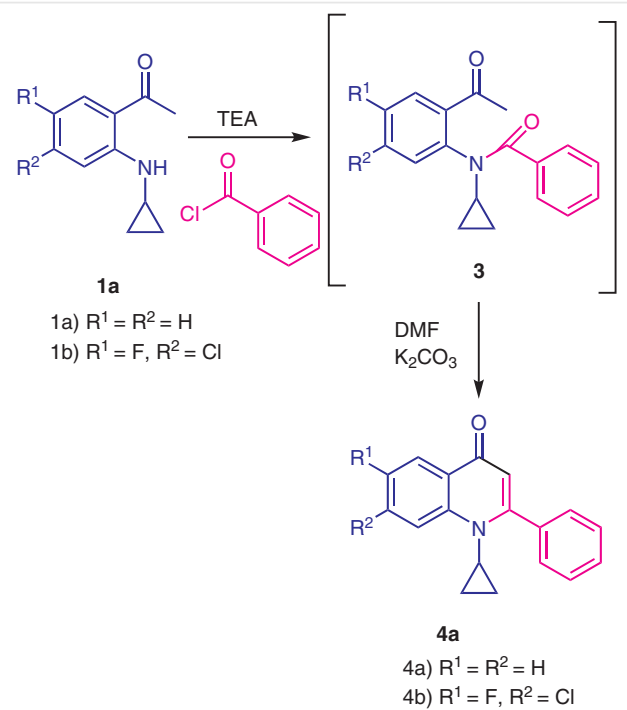

Scheme 3 Synthesis of 2-aryl-2,3-dihydroquinolin-4(1H)-one 4

${ }^{1} \mathrm{H}$ NMR (400 MHz, CDCl $): \delta=1.05-1.09$ (q, $\left.2 \mathrm{H}\right), 1.29-1.34(\mathrm{q}, 2 \mathrm{H})$, 3.34-3.39 (m, $1 \mathrm{H}), 6.20(\mathrm{~d}, J=7.92 \mathrm{~Hz}, 1 \mathrm{H}), 7.66(\mathrm{~d}, J=7.92 \mathrm{~Hz}, 1 \mathrm{H})$, $7.97(\mathrm{~d}, J=5.92 \mathrm{~Hz}, 1 \mathrm{H}), 8.14(\mathrm{~d}, J=9.12 \mathrm{~Hz}, 1 \mathrm{H})$.

${ }^{13} \mathrm{C}$ NMR $\left(100 \mathrm{MHz}, \mathrm{CDCl}_{3}\right): \delta=8.27,33.83,109.72,112.69,118.52$, $126.42,138.23,142.73,153.55,156.05,176.76$.

Anal. Calcd for $\mathrm{C}_{12} \mathrm{H}_{9} \mathrm{ClFNO}$ : C, 60.65; $\mathrm{H}, 3.82 ; \mathrm{N}, 5.89$; Found: $\mathrm{C}$, $60.66 ; \mathrm{H}, 3.80 ; \mathrm{N}, 5.90$.

\section{6-Chloro-1-cyclopropyl-1H-quinolin-4-one (2c)}

Yield: $445 \mathrm{mg}$ (85\%); white solid; $\mathrm{mp} 168.4-174.1{ }^{\circ} \mathrm{C}$.

IR (KBr): 3075, 3012, 1630, 1582, 1473, 1287, 1144, $823 \mathrm{~cm}^{-1}$.

${ }^{1} \mathrm{H}$ NMR $\left(300 \mathrm{MHz}, \mathrm{CDCl}_{3}\right): \delta=1.03-1.09(\mathrm{q}, 2 \mathrm{H}), 1.26-1.33(\mathrm{q}, 2 \mathrm{H})$, $3.35-3.42(\mathrm{~m}, 1 \mathrm{H}), 6.24(\mathrm{~d}, J=7.8 \mathrm{~Hz}, 1 \mathrm{H}), 7.60\left(\mathrm{dd}, J_{1}=2.4\right.$, $\left.J_{2}=2.4 \mathrm{~Hz}, 1 \mathrm{H}\right), 7.67(\mathrm{~d}, J=7.8 \mathrm{~Hz}, 1 \mathrm{H}), 7.85(\mathrm{~d}, J=9.0 \mathrm{~Hz}, 1 \mathrm{H}), 8.39$ $(\mathrm{d}, J=2.4 \mathrm{~Hz}, 1 \mathrm{H})$.

${ }^{13} \mathrm{C}$ NMR $\left(75 \mathrm{MHz}, \mathrm{CDCl}_{3}\right): \delta=8.31,33.80,110.25,118.10,126.09$, 127.77, 130.08, 132.27, 140.02, 142.68, 177.6.

Anal. Calcd for $\mathrm{C}_{12} \mathrm{H}_{10}$ ClNO: C, 65.61; $\mathrm{H}, 4.59$; N, 6.38; Found: C, 65.59; $\mathrm{H}, 4.57 ; \mathrm{N}, 6.40$.

\section{1-Cyclopropyl-7-methoxy-1H-quinolin-4-one (2d)}

Yield: $419 \mathrm{mg}$ (80\%); yellow solid; $\mathrm{mp} 78.1-82.4{ }^{\circ} \mathrm{C}$.

IR (KBr): 3010, 1614, 1569, 1460, 1264, 1016, $827 \mathrm{~cm}^{-1}$.

${ }^{1} \mathrm{H}$ NMR $\left(300 \mathrm{MHz}, \mathrm{CDCl}_{3}\right): \delta=1.05-1.08(\mathrm{q}, 2 \mathrm{H}), 1.23-1.28(\mathrm{q}, 2 \mathrm{H})$, $3.30-3.36(\mathrm{~m}, 1 \mathrm{H}), 3.94(\mathrm{~s}, 3 \mathrm{H}), 6.17(\mathrm{~d}, J=7.8 \mathrm{~Hz}, 1 \mathrm{H}), 6.97\left(\mathrm{dd}, J_{1}=\right.$ 2.4, $\left.J_{2}=2.1 \mathrm{~Hz}, 1 \mathrm{H}\right), 7.62(\mathrm{~d}, J=8.1 \mathrm{~Hz}, 1 \mathrm{H}), 8.34(\mathrm{~d}, J=9.0 \mathrm{~Hz}, 1 \mathrm{H})$.

${ }^{13} \mathrm{C}$ NMR $\left(75 \mathrm{MHz}, \mathrm{CDCl}_{3}\right): \delta=8.21,33.60,55.62,99.03,109.88$, $112.36,121.03,128.69,142.39,143.35,162.65,177.92$.

Anal. Calcd for $\mathrm{C}_{13} \mathrm{H}_{13} \mathrm{NO}_{2}$ : C, 72.54; $\mathrm{H}, 6.09 ; \mathrm{N}, 6.51$; Found: C, 72.55; $\mathrm{H}, 6.07 ; \mathrm{N}, 6.53$.

\section{1-Cyclopropyl-6-methyl-1H-quinolin-4-one (2e)}

Yield: $421 \mathrm{mg}(80 \%)$; yellow solid; mp $96.4-100.7^{\circ} \mathrm{C}$.

IR (KBr): 3032, 3008, 1633, 1604, 1582, 1488, 1341, 1296, 1154, 835 $\mathrm{cm}^{-1}$. 
${ }^{1} \mathrm{H}$ NMR (300 MHz, $\mathrm{CDCl}_{3}$ ): $\delta=1.05-1.07$ (q, $\left.2 \mathrm{H}\right), 1.23-1.27(\mathrm{q}, 2 \mathrm{H}$ ), $2.47(\mathrm{~s}, 3 \mathrm{H}), 3.35-3.40(\mathrm{~m}, 1 \mathrm{H}), 6.21(\mathrm{~d}, J=7.8 \mathrm{~Hz}, 1 \mathrm{H}), 7.49(\mathrm{~d}, J=$ $7.8 \mathrm{~Hz}, 1 \mathrm{H}), 7.65$ (d, J = 7.8 Hz, $1 \mathrm{H}), 7.80$ (d, J = 8.7 Hz, $1 \mathrm{H}), 8.23$ (s, $1 \mathrm{H})$.

${ }^{13} \mathrm{C}$ NMR $\left(75 \mathrm{MHz}, \mathrm{CDCl}_{3}\right): \delta=8.16,20.95,33.63,109.65,116.17$, $126.11,126.65,133.42,133.70,139.65,142.13,178.29$.

Anal. Calcd for $\mathrm{C}_{13} \mathrm{H}_{13} \mathrm{NO}$ : C, 78.36; H, 6.58; N, 7.03; Found: C, 78.34; $\mathrm{H}, 6.59 ; \mathrm{N}, 7.04$.

\section{6-Bromo-1-cyclopropyl-1H-quinolin-4-one (2f)}

Yield: $488 \mathrm{mg}$ (94\%); yellow solid; $\mathrm{mp} 166.3-169.8^{\circ} \mathrm{C}$.

IR (KBr):3075, 3010, 1630, 1580, 1469, $822 \mathrm{~cm}^{-1}$.

${ }^{1} \mathrm{H}$ NMR (300 MHz, $\mathrm{CDCl}_{3}$ ): $\delta=1.03-1.08(\mathrm{q}, 2 \mathrm{H}), 1.25-1.32(\mathrm{q}, 2 \mathrm{H})$, $3.34-3.41(\mathrm{~m}, 1 \mathrm{H}), 6.22(\mathrm{~d}, J=8.0 \mathrm{~Hz}, 1 \mathrm{H}), 7.67(\mathrm{~d}, J=7.8 \mathrm{~Hz}, 1 \mathrm{H})$, 7.75-7.81 $(2 \mathrm{H}, \mathrm{m}), 8.55(\mathrm{~d}, J=1.8 \mathrm{~Hz}, 1 \mathrm{H})$.

${ }^{13} \mathrm{C}$ NMR $\left(75 \mathrm{MHz}, \mathrm{CDCl}_{3}\right): \delta=8.29,33.75,110.32,117.69,118.31$, $128.02,129.22,134.93,140.35,142.72,176.89$.

Anal. Calcd for $\mathrm{C}_{12} \mathrm{H}_{10}$ BrNO: C, 54.57; H, 3.82; N, 5.30; Found: C, 54.56; H, 3.79; N, 5.32.

\section{6,8-Dichloro-1-cyclopropyl-1H-quinolin-4-one (2g)}

Yield: $364 \mathrm{mg}$ (70\%); pale-yellow solid; mp $127.4-132.8^{\circ} \mathrm{C}$.

IR (KBr): 3068, 1633, 1623, 1457, 1336, 1270, $820 \mathrm{~cm}^{-1}$.

${ }^{1} \mathrm{H}$ NMR (300 MHz, $\mathrm{CDCl}_{3}$ ): $\delta=0.91-0.96(\mathrm{q}, 2 \mathrm{H}), 1.17-1.24(\mathrm{q}, 2 \mathrm{H})$, 4.04-4.11 (m, $1 \mathrm{H}), 6.21(\mathrm{~d}, J=8.1 \mathrm{~Hz}, 1 \mathrm{H}), 7.68(\mathrm{~d}, J=2.4 \mathrm{~Hz}, 1 \mathrm{H})$, $7.73(\mathrm{~d}, J=8.1 \mathrm{~Hz}, 1 \mathrm{H}), 8.31(\mathrm{~d}, J=2.4 \mathrm{~Hz}, 1 \mathrm{H})$.

${ }^{13} \mathrm{C}$ NMR $\left(75 \mathrm{MHz}, \mathrm{CDCl}_{3}\right): \delta=11.52,38.96,110.57,123.63,125.60$, $129.81,130.60,135.16,138.28,146.10,176.37$.

Anal. Calcd for $\mathrm{C}_{12} \mathrm{H}_{9} \mathrm{Cl}_{2} \mathrm{NO}$ : C, 56.72; $\mathrm{H}, 3.57$; N, 5.51; Found: C, 56.69; $\mathrm{H}, 3.58 ; \mathrm{N}, 5.48$.

\section{6-Chloro-1-cyclohexyl-1H-quinolin-4-one (2h)}

Yield: $436 \mathrm{mg}$ (84\%); white solid; $\mathrm{mp} 126.8-131.2{ }^{\circ} \mathrm{C}$.

IR (KBr):3061, 2929, 2861, 1621, 1587, 1479, 1353, 1326, 1210, 1170 , $827,805 \mathrm{~cm}^{-1}$.

${ }^{1} \mathrm{H} \mathrm{NMR}\left(300 \mathrm{MHz}, \mathrm{CDCl}_{3}\right): \delta=1.23-1.36(\mathrm{~m}, 1 \mathrm{H}), 1.48-1.52(\mathrm{~m}, 2 \mathrm{H})$, 1.68-1.76 (m, $2 \mathrm{H}), 1.83-1.87$ (m, $1 \mathrm{H}), 2.00-2.05$ (m, $1 \mathrm{H}), 2.11-2.15$ $(\mathrm{m}, 2 \mathrm{H}), 4.29-4.36(\mathrm{~m}, 1 \mathrm{H}), 6.30(\mathrm{~d}, J=8.1 \mathrm{~Hz}, 1 \mathrm{H}), 7.47(\mathrm{~d}, J=$ $9.0 \mathrm{~Hz}, 1 \mathrm{H}), 7.57\left(\mathrm{dd}, J_{1}=2.1, J_{2}=2.4 \mathrm{~Hz}, 1 \mathrm{H}\right), 7.71(\mathrm{~d}, J=8.1 \mathrm{~Hz}, 1 \mathrm{H})$, $8.46(\mathrm{~d}, J=2.1 \mathrm{~Hz}, 1 \mathrm{H})$.

${ }^{13} \mathrm{C}$ NMR $\left(75 \mathrm{MHz}, \mathrm{CDCl}_{3}\right): \delta=25.41,26.00,32.76,58.93,110.35$, $116.56,126.65,128.64,129.59,132.25,138.25,138.53,176.53$.

Anal. Calcd for $\mathrm{C}_{15} \mathrm{H}_{16} \mathrm{ClNO}$ : C, 68.83; $\mathrm{H}, 6.16$; N, 5.35; Found: C, 68.85; H, 6.17; N, 5.32 .

\section{1-Cyclohexyl-1H-quinolin-4-one (2i)}

Yield: $428 \mathrm{mg}$ (82\%); white solid; $\mathrm{mp} 145.3-148.4^{\circ} \mathrm{C}$.

IR (KBr): 3080, 2934, 2860, 1623, 1605, 1581, 1485, 1359, 1209, 1175 , $833 \mathrm{~cm}^{-1}$.

${ }^{1} \mathrm{H}$ NMR (300 MHz, $\left.\mathrm{CDCl}_{3}\right): \delta=1.23-1.32(\mathrm{~m}, 1 \mathrm{H}), 1.49-1.57(\mathrm{~m}, 2 \mathrm{H})$, 1.69-1.77 (m, $2 \mathrm{H}), 1.83-1.87$ ( $\mathrm{m}, 1 \mathrm{H}), 2.00-2.04$ (m, $2 \mathrm{H}), 2.12-2.16$ (m, $2 \mathrm{H}), 4.35-4.43(\mathrm{~m}, 1 \mathrm{H}), 6.31(\mathrm{~d}, J=7.8 \mathrm{~Hz}, 1 \mathrm{H}), 7.35(\mathrm{t}, J=7.5 \mathrm{~Hz}$, $1 \mathrm{H}), 7.53(\mathrm{~d}, J=8.7 \mathrm{~Hz}, 1 \mathrm{H}), 7.64(\mathrm{t}, J=7.5 \mathrm{~Hz}, 1 \mathrm{H}), 7.72(\mathrm{~d}, J=8.1 \mathrm{~Hz}$, $1 \mathrm{H}), 8.49(\mathrm{~d}, J=7.8 \mathrm{~Hz}, 1 \mathrm{H})$.

${ }^{13} \mathrm{C}$ NMR $\left(75 \mathrm{MHz}, \mathrm{CDCl}_{3}\right): \delta=25.53,26.10,32.86,58.54,110.20$, $114.63,123.44,127.51,127.70,132.09,138.10,140.18,177.85$.
Anal. Calcd for $\mathrm{C}_{15} \mathrm{H}_{17} \mathrm{NO}$ : C, 79.26; H, 7.54; N, 6.16; Found: C, 79.24; $\mathrm{H}, 7.55 ; \mathrm{N}, 6.14$.

\section{1-Cyclopentyl-1H-quinolin-4-one (2j)}

Yield: $440 \mathrm{mg}$ (84\%); yellow solid; $\mathrm{mp} 104.6-107.8^{\circ} \mathrm{C}$.

IR (KBr): 3067, 2963, 1625, 1606, 1579, 1488, 1354, 1210, 1179, 838 $\mathrm{cm}^{-1}$.

${ }^{1} \mathrm{H}$ NMR $\left(300 \mathrm{MHz}, \mathrm{CDCl}_{3}\right): \delta=1.80-1.93(\mathrm{~m}, 6 \mathrm{H}), 2.25-2.29(\mathrm{~m}, 2 \mathrm{H})$, $4.94(\mathrm{~m}, 1 \mathrm{H}), 6.31(\mathrm{~d}, J=7.8 \mathrm{~Hz}, 1 \mathrm{H}), 7.36(\mathrm{t}, J=7.2 \mathrm{~Hz}, 1 \mathrm{H}), 7.60-$ $7.70(\mathrm{~m}, 3 \mathrm{H}), 8.48(\mathrm{~d}, J=7.8 \mathrm{~Hz}, 1 \mathrm{H})$.

${ }^{13} \mathrm{C}$ NMR $\left(75 \mathrm{MHz}, \mathrm{CDCl}_{3}\right): \delta=24.05,32.29,60.61,110.10,115.43$, 123.49, 127.27, 127.57, 132.01, 138.22, 140.73, 177.94.

Anal. Calcd for $\mathrm{C}_{14} \mathrm{H}_{15} \mathrm{NO}$ : C, 78.84; H, 7.09; N, 6.57; Found: C, 78.85; $\mathrm{H}, 7.10 ; \mathrm{N}, 6.54$.

\section{6-Chloro-1-cyclopentyl-1H-quinolin-4-one (2k)}

Yield: $448 \mathrm{mg}$ (86\%); white solid; $\mathrm{mp} 141.7-143.2{ }^{\circ} \mathrm{C}$.

IR (KBr): 3079, 2954, 2877, 1626, 1585, 1483, 1206, 1008, 845, 823 $\mathrm{cm}^{-1}$.

${ }^{1} \mathrm{H}$ NMR $\left(300 \mathrm{MHz}, \mathrm{CDCl}_{3}\right): \delta=1.85-1.93(\mathrm{~m}, 6 \mathrm{H}), 2.25-2.27(\mathrm{~m}, 2 \mathrm{H})$, 4.87-4.88 (m, $1 \mathrm{H}), 6.29$ (d, J = 8.1 Hz, $1 \mathrm{H}), 7.54-7.61(\mathrm{~m}, 2 \mathrm{H}), 7.65$ $(\mathrm{d}, J=7.8 \mathrm{~Hz}, 1 \mathrm{H}), 8.45(\mathrm{~d}, J=1.5 \mathrm{~Hz}, 1 \mathrm{H})$.

${ }^{13} \mathrm{C}$ NMR $\left(75 \mathrm{MHz}, \mathrm{CDCl}_{3}\right): \delta=24.03,32.28,60.98,110.36,117.31$, 126.56, 128.63, 129.78, 132.25, 138.36, 139.15 .

Anal. Calcd for $\mathrm{C}_{14} \mathrm{H}_{14} \mathrm{ClNO}$ : C, 67.88; H, 5.70; N, 5.65; Found: C, 67.86; H, 5.67; N, 5.67.

\section{1-Methyl-1H-quinolin-4-one (21)}

Yield: $373 \mathrm{mg}$ (70\%); white solid; $\mathrm{mp} 144.6-148.1{ }^{\circ} \mathrm{C}$. IR (KBr): 3061, 3017, 1625, 1576, 1493, 1237, $759 \mathrm{~cm}^{-1}$. ${ }^{1} \mathrm{H}$ NMR $\left(400 \mathrm{MHz}, \mathrm{CDCl}_{3}\right): \delta=3.81(\mathrm{~s}, 3 \mathrm{H}), 6.28(\mathrm{~d}, J=7.6 \mathrm{~Hz}, 1 \mathrm{H})$, $7.41(\mathrm{t}, J=3.4 \mathrm{~Hz}, 2 \mathrm{H}), 7.52(\mathrm{~d}, J=7.6 \mathrm{~Hz}, 1 \mathrm{H}), 7.71(\mathrm{t}, J=7.8 \mathrm{~Hz}, 1 \mathrm{H})$, $8.48(\mathrm{~d}, J=8.0 \mathrm{~Hz}, 1 \mathrm{H})$.

${ }^{13} \mathrm{C}$ NMR $\left(100 \mathrm{MHz}, \mathrm{CDCl}_{3}\right): \delta=40.61,110.15,115.23,123.77,127.06$, 127.13, 132.20, 140.67, 143.62, 178.32.

Anal. Calcd for $\mathrm{C}_{10} \mathrm{H}_{9} \mathrm{NO}$ : C, 75.45; H, 5.70; N, 8.80; Found: C, 75.43; $\mathrm{H}$, 5.68; N, 8.81.

\section{1-(4-Chloro-phenyl)-1H-quinolin-4-one (2m)}

Yield: $884 \mathrm{mg}$ (85\%); yellow solid; $\mathrm{mp} 177.2-181.5^{\circ} \mathrm{C}$.

IR (KBr): 3045, 3022, 1622, 1606, 1590, 1476, 1367, 1285, 1236, 760 $\mathrm{cm}^{-1}$.

${ }^{1} \mathrm{H}$ NMR $\left(400 \mathrm{MHz}, \mathrm{CDCl}_{3}\right): \delta=6.38(\mathrm{~d}, J=7.6 \mathrm{~Hz}, 1 \mathrm{H}), 6.98(\mathrm{~d}, J=$ $8.4 \mathrm{~Hz}, 1 \mathrm{H}$ ), 7.40-7.35 (m, $3 \mathrm{H}), 7.59-7.49$ ( $\mathrm{m}, 4 \mathrm{H}), 8.46$ (dd, $J_{1}=0.8$, $\left.J_{2}=0.8 \mathrm{~Hz}, 1 \mathrm{H}\right)$.

${ }^{13} \mathrm{C}$ NMR $\left(100 \mathrm{MHz}, \mathrm{CDCl}_{3}\right): \delta=110.54,116.99,124.10,126.60$, $126.74,129.01,130.61,132.04,135.59,139.81,141.20,142.41$, 178.23 .

Anal. Calcd for $\mathrm{C}_{15} \mathrm{H}_{10} \mathrm{ClNO}$ : C, 70.46; $\mathrm{H}, 3.94 ; \mathrm{N}, 5.48$; Found: C, 70.47; H, 3.92; N, 5.46.

1-(4-Bromo-phenyl)-6-methyl-1H-quinolin-4-one (2n) Yield: $454 \mathrm{mg}$ (88\%); white solid; $\mathrm{mp} 145.3-148.4^{\circ} \mathrm{C}$. IR (KBr): 3021, 1630, 1610, 1583, 1483, 1289, 1201, $823 \mathrm{~cm}^{-1}$. 
${ }^{1} \mathrm{H}$ NMR $\left(400 \mathrm{MHz}, \mathrm{CDCl}_{3}\right): \delta=2.45(\mathrm{~s}, 3 \mathrm{H}), 6.36(\mathrm{~d}, J=7.6 \mathrm{~Hz}, 1 \mathrm{H})$, $6.89(\mathrm{~d}, J=8.8 \mathrm{~Hz}, 1 \mathrm{H}), 7.29(\mathrm{~d}, J=8.4 \mathrm{~Hz}, 2 \mathrm{H}), 7.34\left(\mathrm{dd}, J_{1}=2.0\right.$, $\left.J_{2}=2.0 \mathrm{~Hz}, 1 \mathrm{H}\right), 7.52(\mathrm{~d}, J=7.6 \mathrm{~Hz}, 1 \mathrm{H}), 7.73(\mathrm{~d}, J=8.4 \mathrm{~Hz}, 2 \mathrm{H}), 8.26$ $(\mathrm{s}, 1 \mathrm{H})$.

${ }^{13} \mathrm{C} \mathrm{NMR}\left(100 \mathrm{MHz}, \mathrm{CDCl}_{3}\right): \delta=20.89,110.25,116.91,123.45,126.05$, $126.45,129.24,133.46,133.55,134.17,139.20,140.44,141.99$, 178.18 .

Anal. Calcd for $\mathrm{C}_{16} \mathrm{H}_{12}$ BrNO: C, 61.17; $\mathrm{H}, 3.85$; N, 4.46; Found: C, $61.15 ; \mathrm{H}, 3.87 ; \mathrm{N}, 4.43$.

\section{1-(4-Bromo-phenyl)-1H-quinolin-4-one (20)}

Yield: $806 \mathrm{mg}$ (78\%); yellow solid; mp $198.1-200.1{ }^{\circ} \mathrm{C}$.

IR (KBr): 3043, 3020, 1619, 1591, 1475, 1366, 1283, $1238 \mathrm{~cm}^{-1}$.

${ }^{1} \mathrm{H}$ NMR $\left(400 \mathrm{MHz}, \mathrm{CDCl}_{3}\right): \delta=6.37(\mathrm{~d}, J=7.6 \mathrm{~Hz}, 1 \mathrm{H}), 6.98(\mathrm{~d}, J=$ $8.4 \mathrm{~Hz}, 1 \mathrm{H}), 7.30(\mathrm{~d}, J=8.0 \mathrm{~Hz}, 2 \mathrm{H}), 7.40(\mathrm{t}, J=7.4 \mathrm{~Hz}, 1 \mathrm{H}), 7.53(\mathrm{dd}$, $\left.J_{1}=1.2, J_{2}=1.6 \mathrm{~Hz}, 1 \mathrm{H}\right), 7.55(\mathrm{~d}, J=8.0 \mathrm{~Hz}, 1 \mathrm{H}), 7.75(\mathrm{~d}, J=7.6 \mathrm{~Hz}$, $2 \mathrm{H}), 8.48\left(\mathrm{dd}, J_{1}=0.8, J_{2}=0.8 \mathrm{~Hz}, 1 \mathrm{H}\right)$.

${ }^{13} \mathrm{C}$ NMR $\left(100 \mathrm{MHz}, \mathrm{CDCl}_{3}\right): \delta=110.57,116.98,123.56,124.10$, $126.61,126.75,129.31,132.04,133.62,140.34,141.13,142.31$, 178.21 .

Anal. Calcd for $\mathrm{C}_{15} \mathrm{H}_{10} \mathrm{BrNO}$ : C, 60.02; $\mathrm{H}, 3.36$; N, 4.67; Found: C, 60.03; $\mathrm{H}, 3.34 ; \mathrm{N}, 4.64$.

\section{1-(4-Isopropyl-phenyl)-1H-quinolin-4-one (2p)}

Yield: $893 \mathrm{mg}$ (86\%); yellow solid; $\mathrm{mp} 44.7-46.5^{\circ} \mathrm{C}$.

IR (KBr): 2964, 1620, 1585, 1291, $760 \mathrm{~cm}^{-1}$

${ }^{1} \mathrm{H} \mathrm{NMR}\left(400 \mathrm{MHz}, \mathrm{CDCl}_{3}\right): \delta=1.34(\mathrm{~d}, J=6.8 \mathrm{~Hz}, 6 \mathrm{H}), 3.00-307(\mathrm{~m}$, $1 \mathrm{H}), 6.38(\mathrm{~d}, J=8.0 \mathrm{~Hz}, 1 \mathrm{H}), 7.04(\mathrm{~d}, J=8.4 \mathrm{~Hz}, 1 \mathrm{H}), 7.31(\mathrm{~d}, J=$ $8.4 \mathrm{~Hz}, 2 \mathrm{H}), 7.38(\mathrm{t}, J=7.6 \mathrm{~Hz}, 1 \mathrm{H}), 7.44(\mathrm{~d}, J=8.0 \mathrm{~Hz}, 2 \mathrm{H}), 7.49(\mathrm{t}, J=$ $8.4 \mathrm{~Hz}, 1 \mathrm{H}), 7.61(\mathrm{~d}, J=7.6 \mathrm{~Hz}, 1 \mathrm{H}), 8.48\left(\mathrm{dd}, J_{1}=1.2, J_{2}=1.2 \mathrm{~Hz}, 1 \mathrm{H}\right)$. ${ }^{13} \mathrm{C}$ NMR $\left(100 \mathrm{MHz}, \mathrm{CDCl}_{3}\right): \delta=23.94,33.95,110.11,117.45,123.81$, $126.52,126.60,127.35,128.25,131.77,138.99,141.52,142.93$, $150.49,178.29$.

Anal. Calcd for $\mathrm{C}_{18} \mathrm{H}_{17} \mathrm{NO}$ : C, 82.10; $\mathrm{H}, 6.51 ; \mathrm{N}, 5.32$; Found: $\mathrm{C}, 82.08$; $\mathrm{H}, 6.52 ; \mathrm{N}, 5.30$.

\section{6-Methyl-1-phenyl-1H-quinolin-4-one (2q)}

Yield: $887 \mathrm{mg}$ (85\%); white solid; $\mathrm{mp} 113.2-115.7^{\circ} \mathrm{C}$.

IR (KBr): 3030, 1584, 1486, 1286, 830, 808, 765, $695 \mathrm{~cm}^{-1}$.

${ }^{1} \mathrm{H}$ NMR $\left(300 \mathrm{MHz}, \mathrm{CDCl}_{3}\right): \delta=2.46(\mathrm{~s}, 3 \mathrm{H}), 6.36(\mathrm{~d}, J=7.5 \mathrm{~Hz}, 1 \mathrm{H})$, $6.92(\mathrm{~d}, J=8.7 \mathrm{~Hz}, 1 \mathrm{H}), 7.301(\mathrm{~d}, J=8.4 \mathrm{~Hz}, 1 \mathrm{H}), 7.39(\mathrm{~d}, J=6.3 \mathrm{~Hz}$, $2 \mathrm{H}), 7.56-7.60(\mathrm{~m}, 4 \mathrm{H}), 8.27$ (s, $1 \mathrm{H})$.

${ }^{13} \mathrm{C}$ NMR $\left(75 \mathrm{MHz}, \mathrm{CDCl}_{3}\right): \delta=21.00,110.03,117.34,125.98,126.57$, $127.65,129.53,130.37,133.39,133.99,139.57,141.60,142.50$, 178.35 .

Anal. Calcd for $\mathrm{C}_{16} \mathrm{H}_{13} \mathrm{NO}$ : C, 81.68; $\mathrm{H}, 5.57$; N, 5.95; Found: C, 81.66; $\mathrm{H}, 5.58 ; \mathrm{N}, 5.97$.

\section{6-Bromo-1-phenyl-1H-quinolin-4-one (2r)}

Yield: $827 \mathrm{mg}$ (80\%); yellow solid; $\mathrm{mp} 158-162.8{ }^{\circ} \mathrm{C}$

IR (KBr): 3054, 3043, 1630, 1584, 1471, 1292, 818, $698 \mathrm{~cm}^{-1}$.

${ }^{1} \mathrm{H}$ NMR $\left(300 \mathrm{MHz}, \mathrm{CDCl}_{3}\right): \delta=6.39(\mathrm{~d}, J=7.5 \mathrm{~Hz}, 1 \mathrm{H}), 6.88(\mathrm{~d}, J=$ $9.0 \mathrm{~Hz}, 1 \mathrm{H}), 7.37(\mathrm{~d}, J=7.5 \mathrm{~Hz}, 2 \mathrm{H}), 7.54-7.61(\mathrm{~m}, 5 \mathrm{H}), 8.61(\mathrm{~s}, 1 \mathrm{H})$.

${ }^{13} \mathrm{C}$ NMR $\left(75 \mathrm{MHz}, \mathrm{CDCl}_{3}\right): \delta=110.68,117.85,119.39,127.53,127.94$, $129.25,129.91,130.61,134.96,140.26,141.09,143.03,177.04$.
Anal. Calcd for $\mathrm{C}_{15} \mathrm{H}_{10} \mathrm{BrNO}$ : C, 60.02; $\mathrm{H}, 3.36$; N, 4.67; Found: C, 60.00; $\mathrm{H}, 3.32 ; \mathrm{N}, 4.65$.

\section{6-Chloro-1-phenyl-1H-quinolin-4-one (2s)}

Yield: $832 \mathrm{mg}(80 \%)$; white solid; $\mathrm{mp} 161.5-164.7^{\circ} \mathrm{C}$.

IR (KBr): 3042, 1630, 1590, 1473, 1294, 817, $702 \mathrm{~cm}^{-1}$

${ }^{1} \mathrm{H}$ NMR $\left(300 \mathrm{MHz}, \mathrm{CDCl}_{3}\right): \delta=6.37(\mathrm{~d}, J=7.8 \mathrm{~Hz}, 1 \mathrm{H}), 6.95(\mathrm{~d}, J=$ $9.0 \mathrm{~Hz}, 1 \mathrm{H}), 7.38-7.44(\mathrm{~m}, 3 \mathrm{H}), 7.58-7.62(\mathrm{~m}, 4 \mathrm{H}), 8.44$ (d, $J=2.4 \mathrm{~Hz}$, $1 \mathrm{H})$.

${ }^{13} \mathrm{C}$ NMR $\left(75 \mathrm{MHz}, \mathrm{CDCl}_{3}\right): \delta=110.58,119.20,126.05,127.56,127.65$, $129.90,130.23,130.60,132.28,139.90,141.16,142.97,177.16$

Anal. Calcd for $\mathrm{C}_{15} \mathrm{H}_{10} \mathrm{ClNO}$ : C, 70.46; H, 3.94; N, 5.48; Found: C, 70.47; H, 3.92; N, 5.46 .

\section{1-(4-Nitro-phenyl)-1H-quinolin-4-one (2t)}

Yield: $441 \mathrm{mg}$ (85\%); yellow solid; $\mathrm{mp} 164.1-167.8{ }^{\circ} \mathrm{C}$. IR (KBr): 3354, 1682, 1594, 1504, 1330, 1304, 1111, $740 \mathrm{~cm}^{-1}$. ${ }^{1} \mathrm{H}$ NMR $\left(400 \mathrm{MHz}, \mathrm{CDCl}_{3}\right): \delta=6.43(\mathrm{~d}, J=7.6 \mathrm{~Hz}, 1 \mathrm{H}), 6.99(\mathrm{~d}, J=$ $8.4 \mathrm{~Hz}, 1 \mathrm{H}), 7.42(\mathrm{t}, J=7.2 \mathrm{~Hz}, 1 \mathrm{H}), 7.54-7.58(\mathrm{~m}, 2 \mathrm{H}), 7.65(\mathrm{~d}, J=$ $8.8 \mathrm{~Hz}, 2 \mathrm{H}), 8.49(\mathrm{~d}, J=8.8 \mathrm{~Hz}, 3 \mathrm{H})$.

${ }^{13} \mathrm{C}$ NMR $\left(100 \mathrm{~Hz}, \mathrm{CDCl}_{3}\right): \delta=111.21,116.52,124.59,125.82,126.62$, $127.05,128.73,132.37,140.57,141.65,146.58,147.94,178.10$.

Anal. Calcd for $\mathrm{C}_{15} \mathrm{H}_{10} \mathrm{~N}_{2} \mathrm{O}_{3}$ : C, 67.67; H, 3.79; N, 10.52; Found: C, $67.62 ; \mathrm{H}, 3.74 ; \mathrm{N}, 10.49$.

\section{1-(3-Methoxy-phenyl)-1H-quinolin-4-one (2u)}

Yield: $426 \mathrm{mg}$ (82\%); yellow solid; mp $163.5-167.1{ }^{\circ} \mathrm{C}$.

IR (KBr): 3056, 2840, 1631, 1585, 1225, 1032, $699 \mathrm{~cm}^{-1}$.

${ }^{1} \mathrm{H}$ NMR $\left(400 \mathrm{MHz}, \mathrm{CDCl}_{3}\right): \delta=3.86(\mathrm{~s}, 3 \mathrm{H}), 6.36(\mathrm{~d}, J=8.0 \mathrm{~Hz}, 1 \mathrm{H})$, $6.92(\mathrm{~d}, J=2.0 \mathrm{~Hz}, 1 \mathrm{H}), 6.98(\mathrm{t}, J=7.6 \mathrm{~Hz}, 1 \mathrm{H}), 7.04-7.10(\mathrm{~m}, 2 \mathrm{H})$, $7.36(\mathrm{t}, J=7.4 \mathrm{~Hz}, 1 \mathrm{H}), 7.47-7.52(\mathrm{~m}, 2 \mathrm{H}), 7.60(\mathrm{~d}, J=7.6 \mathrm{~Hz}, 2 \mathrm{H})$, $8.46(\mathrm{~d}, J=7.2 \mathrm{~Hz}, 1 \mathrm{H})$

${ }^{13} \mathrm{C}$ NMR $\left(100 \mathrm{~Hz}, \mathrm{CDCl}_{3}\right): \delta=55.65,110.17,113.25,115.21,117.35$, $119.59,123.87,126.56,131.02,131.85,141.29,142.39,142.59$, $161.00,178.29$.

Anal. Calcd for $\mathrm{C}_{16} \mathrm{H}_{13} \mathrm{NO}_{2}$ : C, 76.48; H, 5.21; N, 5.57; Found: C, 76.49; $\mathrm{H}, 5.23 ; \mathrm{N}, 5.56$

\section{Preparation of 1-Cyclopropyl-2-phenyl-1H-quinolin-4-one (4a)}

To a mixture of 1-(2-cyclopropylaminophenyl)ethanone $\mathbf{1}$ (1.0 g, 5.71 $\mathrm{mmol})$ and triethylamine $(2.88 \mathrm{~g}, 28.5 \mathrm{mmol})$ in THF $(10 \mathrm{~mL})$ at $25^{\circ} \mathrm{C}$, benzoyl chloride $(0.802 \mathrm{~g}, 5.71 \mathrm{mmol})$ was added and the mixture was heated to reflux for $4 \mathrm{~h}$. After completion of reaction (as monitored by TLC), the THF was removed under reduced pressure and potassium carbonate $(2.36 \mathrm{~g}, 17.10 \mathrm{mmol})$ and $\mathrm{DMF}(10 \mathrm{~mL})$ were added at $25^{\circ} \mathrm{C}$. The reaction mixture was then heated to $100{ }^{\circ} \mathrm{C}$ until completion of the reaction (monitored by TLC). The reaction mixture was then poured into water and extracted with EtOAc $(3 \times 10 \mathrm{~mL})$. The combined organic phases were dried over sodium sulfate, filtered and concentrated under reduced pressure. The residue was purified by silica gel column chromatography eluting with EtOAc/n-hexane to obtain 4a.

\section{1-Cyclopropyl-2-phenyl-1H-quinolin-4-one (4a)}

Yield: $650 \mathrm{mg}$ (87\%); white solid; $\mathrm{mp} 170.1-172.4{ }^{\circ} \mathrm{C}$.

IR (KBr): 3049, 3009, 1617, 1597, 1478, 1462, 1408, 1311, 1271, 1138, $1043,775,758,709 \mathrm{~cm}^{-1}$. 
${ }^{1} \mathrm{H}$ NMR $\left(400 \mathrm{MHz}, \mathrm{CDCl}_{3}\right): \delta=0.57-0.58(\mathrm{q}, 2 \mathrm{H}), 0.91-0.95(\mathrm{q}, 2 \mathrm{H})$, 3.32-3.35 (m, $1 \mathrm{H}), 6.32(\mathrm{~s}, 1 \mathrm{H}), 7.38(\mathrm{t}, J=7.6 \mathrm{~Hz}, 1 \mathrm{H}), 7.47-7.54(\mathrm{~m}$, $5 \mathrm{H}), 7.68-7.72(\mathrm{~m}, 1 \mathrm{H}), 7.96(\mathrm{~d}, J=8.8 \mathrm{~Hz}, 1 \mathrm{H}), 8.44\left(\mathrm{dd}, J_{1}=1.2, J_{2}=\right.$ $1.2 \mathrm{~Hz}, 1 \mathrm{H})$.

${ }^{13} \mathrm{C}$ NMR $\left(100 \mathrm{MHz}, \mathrm{CDCl}_{3}\right): \delta=12.92,32.37,113.32,117.82,123.53$, $126.41,126.77,128.36,128.52,129.20,131.67,136.96,143.12$, 155.45, 178.19.

Anal. Calcd for $\mathrm{C}_{18} \mathrm{H}_{15} \mathrm{NO}$ : C, 82.73; $\mathrm{H}, 5.79$; N, 5.36; Found: C, 82.74; H, 5.76; N, 5.34 .

\section{7-Chloro-1-cyclopropyl-6-fluoro-2-phenyl-1H-quinolin-4-one (4b)}

Yield: $575 \mathrm{mg}$ (84\%); white solid; $\mathrm{mp} 209.2-211.5^{\circ} \mathrm{C}$.

IR (KBr): 3073, 1631, 1609, 1468, 1271, 986, $840 \mathrm{~cm}^{-1}$.

${ }^{1} \mathrm{H}$ NMR (400 MHz, $\mathrm{CDCl}_{3}$ ): $\delta=0.58-0.59$ (q, $\left.2 \mathrm{H}\right), 0.95-1.00(\mathrm{q}, 2 \mathrm{H})$, 3.29-3.32 (m, $1 \mathrm{H}), 6.26(\mathrm{~s}, 1 \mathrm{H}), 7.49(\mathrm{~m}, 5 \mathrm{H}), 8.03(\mathrm{~d}, J=6.0 \mathrm{~Hz}, 1 \mathrm{H})$, $8.13(\mathrm{~d}, J=8.8 \mathrm{~Hz}, 1 \mathrm{H})$.

${ }^{13} \mathrm{C}$ NMR $\left(100 \mathrm{MHz}, \mathrm{CDCl}_{3}\right): \delta=12.96,32.66,112.25,112.47,113.00$, $120.18,125.99,126.19,126.66,126.72,128.29,128.67,129.53$, 136.38, 139.74, 153.42, 155.85, 155.89, 176.58, 176.60.

Anal. Calcd for $\mathrm{C}_{18} \mathrm{H}_{13}$ ClFNO: C, 68.91; $\mathrm{H}, 4.18$; N, 4.46; Found: $\mathrm{C}$, $68.90 ; \mathrm{H}, 4.17 ; \mathrm{N}, 4.45$.

\section{Acknowledgment}

We would like to thank Suven Life Sciences for providing analytical facilities and acknowledge CEO Mr Jasti for permission to publish this work.

\section{Supporting Information}

Supporting information for this article is available online at https://doi.org/10.1055/s-0037-1610388.

\section{References}

(1) Pozharskii, A. F.; Soldatenkov, A. T.; Katritzky, A. R. Heterocycles in Life and Society; Wiley: Chichester, UK, 1997, 135.

(2) (a) Mitscher, L. A. Chem. Rev. 2005, 105, 559. (b) Cheng, G.; Hao, H.; Dai, M.; Liu, Z.; Yuan, Z. Eur. J. Med. Chem. 2013, 66, 555.

(3) (a) Chen, Y.-L.; Fang, K.-C.; Sheu, J.-Y.; Hsu, S.-L.; Tzeng, C.-C. J. Med. Chem. 2001, 44, 2374. (b) Asahina, Y.; Iwase, K.; Iinuma, F.; Hosaka, M.; Ishizaki, T. J. Med. Chem. 2005, 48, 3194. (c) Odagiri, T.; Inagaki, H.; Sugimoto, Y.; Nagamochi, M.; Miyauchi, R. N.; Kuroyanagi, J.; Kitamura, T.; Komoriya, S.; Takahashi, H. J. Med. Chem. 2013, 56, 1974.

(4) (a) Li, L.; Wang, H.-K.; Kuo, S.-C.; Wu, T.-S.; Mauger, A.; Lin, C. M.; Hamel, E.; Lee, K.-H. J. Med. Chem. 1994, 37, 3400. (b) Nakamura, S.; Kozuka, M.; Bastow, K. F.; Tokuda, H.; Nishino, H.; Suzuki, M.; Tatsuzaki, J.; Natschke, S. L. M.; Kuo, S.-C.; Lee, K.-H. Bioorg. Med. Chem. 2005, 13, 4396. (c) Chang, Y.-H.; Hsu, M.-H.; Wang, S.-H.; Huang, L.-J.; Qian, K.; Morris-Natschke, S. L.; Hamel, E.; Kuo, S.-C.; Lee, K.-H. J. Med. Chem. 2009, 52, 4883.

(5) (a) Cross, R. M.; Monastyrskyi, A.; Mutka, T. S.; Burrows, J. N.; Kyle, D. E.; Manetsch, R. J. Med. Chem. 2010, 53, 7076. (b) Cross, R. M.; Namelikonda, N. K.; Mutka, T. S.; Luong, L.; Kyle, D. E.; Manetsch, R. J. Med. Chem. 2011, 54, 8321. (c) Zhang, Y.; Clark, J. A.; Connelly, M. C.; Zhu, F.; Min, J.; Guiguemde, W. A.; Pradhan,
A.; Iyer, L.; Furimsky, A.; Gow, J.; Parman, T.; Mazouni, F. E.; Phillips, M. A.; Kyle, D. E.; Mirsalis, J.; Guy, R. K. J. Med. Chem. 2012, 55, 4205.

(6) Edmont, D.; Rocher, R.; Plisson, C.; Chenault, J. Bioorg. Med. Chem. Lett. 2000, 10, 1831.

(7) Lucero, B. d'. A.; Gomes, C. R. B.; Frugulhetti, I. C. de P. P.; Faro, L. V.; Alvarenga, L.; Souza, M. C. B. V.; de Souza, T. M. L.; Ferreira, V. F. Bioorg. Med. Chem. Lett. 2006, 16, 1010.

(8) (a) Cecchetti, V.; Parolin, C.; Moro, S.; Pecere, T.; Filipponi, E.; Calistri, A.; Tabarrini, O.; Gatto, B.; Palumbo, M.; Fravolini, A.; Palu, G. J. Med. Chem. 2000, 43, 3799. (b) Sato, M.; Motomura, T.; Aramaki, H.; Matsuda, T.; Yamashita, M.; Ito, Y.; Kawakami, H.; Matsuzaki, Y.; Watanabe, W.; Yamataka, K.; Ikeda, S.; Kodama, E.; Matsuoka, M.; Shinkai, H. J. Med. Chem. 2006, 49, 1506. (c) Pasquini, S.; Mugnaini, C.; Tintori, C.; Botta, M.; Trejos, A.; Arvela, R. K.; Larhed, M.; Witvrouw, M.; Michiels, M.; Christ, F.; Debyser, Z.; Corelli, F. J. Med. Chem. 2008, 51, 5125.

(9) (a) Reitsema, R. H. Chem. Rev. 1948, 43, 43. (b) López, S. E.; Rebollo, O.; Salazar, J.; Charris, J. E.; Yánez, C. J. Fluorine Chem. 2003, 120, 71. (c) Boteva, A. A.; Krasnykh, O. P. Chem. Heterocycl. Compd. 2009, 45, 757. (d) Romek, A.; Opatz, T. Eur. J. Org. Chem. 2010, 5841. (e) Liu, Q.-L.; Li, Q.-L.; Fei, X.-D.; Zhu, Y.-M. ACS Comb. Sci. 2011, 13, 19. (f) Zhao, J.; Zhao, Y.; Fu, H. Org. Lett. 2012, 14, 2710. (g) Iaroshenko, V. O.; Knepper, I.; Zahid, M.; Dudkin, S.; Kuzora, R.; Villinger, A.; Langer, P. Org. Biomol. Chem. 2012, 10, 2955. (h) Shao, J.; Huang, X.; Hong, X.; Liu, B.; Xu, B. Synthesis 2012, 44, 1798. (i) Victor, N. J.; Muraleedharan, K. M. Adv. Synth. Catal. 2014, 356, 3600. (j) Ji, X.; Wang, Z.; Tan, M.; Huang, H.; Deng, G. J. Asian J. Org. Chem. 2018, 7, 711. (k) Wang, D.; Sun, P.; Jia, p.; Peng, J.; Yue, Y.; Chen, C. Synthesis 2017, 49, 1851. (1) Xu, X.; Zhang, X. Org. Lett. 2017, 19, 4984.

(10) Camps, R. Ber. Dtsch. Chem. Ges. 1899, 32, 3228.

(11) (a) Li, J. J. Name Reactions: A Collection of Detailed Reaction Mechanisms 2nd Ed; Springer-Verlag: Berlin, 2003, 81. (b) Zewge, D.; Chen, C.-Y.; Deer, C.; Dormer, P. G.; Hughes, D. L. J. Org. Chem. 2007, 72, 4276.

(12) Gould, R. G.; Jacobs, W. A. J. Am. Chem. Soc. 1939, 61, 2890.

(13) (a) Niementowski, S. Ber. Dtsch. Chem. Ges. 1894, 27, 1394. (b) Fuson, R. C.; Burness, D. M. J. Am. Chem. Soc. 1946, 68, 1270. (c) Son, J. K.; Kim, S. I.; Jahng, Y. Heterocycles 2001, 55, 1981.

(14) (a) Kalinin, V. N.; Sbostakovsky, M. V.; Ponomaryov, A. B. Tetrahedron Lett. 1992, 33, 373. (b) Torii, S.; Okumoto, H.; Xu, L. H.; Sadakane, M.; Shostakovsky, M. V.; Ponomaryov, A. B.; Kalinin, V. N. Tetrahedron 1993, 49, 6773. (c) Haddad, N.; Tan, J.; Farina, V. J. Org. Chem. 2006, 71, 5031. (d) Huang, J.; Chen, Y.; King, A. O.; Dilmeghani, M.; Larsen, R. D.; Faul, M. M. Org. Lett. 2008, 10, 2609. (e) Zhao, T.; Xu, B. Org. Lett. 2010, 12, 212. (f) Takahashi, I.; Morita, F.; Kusagaya, S.; Fukaya, H.; Kitagawa, O. Tetrahedron: Asymmetry 2012, 23, 1657. (g) Iaroshenko, V. O.; Knepper, I.; Zahid, M.; Kuzora, R.; Dudkin, S.; Villinger, A.; Langer, P. Org. Biomol. Chem. 2012, 10, 2955. (h) Fei, X.-D.; Zhou, Z.; Li, W.; Zhu, Y.-M.; Shen, J.-K. Eur. J. Org. Chem. 2012, 3001. (i) Iaroshenko, V. O.; Zahid, M.; Mkrtchyan, S.; Gevorgyan, A.; Altenburger, K.; Knepper, I.; Villinger, A.; Sosnovskikh, V. Y.; Langer, P. Tetrahedron 2013, 69, 2309. (j) Wang, Y.; Liang, H.; Chen, C.; Wang, D.; Peng, J. Synthesis 2015, 47, 1851.

(15) (a) Jones, C. P.; Anderson, K. W.; Buchwald, S. L. J. Org. Chem. 2007, 72, 7968. (b) Bernini, R.; Cacchi, S.; Fabrizi, G.; Sferrazza, A. Synthesis 2009, 1209.

(16) Seppänen, O.; Muuronen, M.; Helaja, J. Eur. J. Org. Chem. 2014, 4044.

(17) Radl, S.; Dax, S. Curr. Med. Chem. 1994, 1, 262. 
(18) (a) Alvarez, M.; Salas, M.; Rigat, L.; de Veciana, A.; Joule, J. A. J. Chem. Soc., Perkin Trans. 1 1992, 351. (b) Bichovski, P.; Haas, T. M.; Kratzert, D.; Streuff, J. Chem. Eur. J. 2015, 21, 2339. (c) Biswas, K.; Peterkin, T. A. N.; Bryan, M. C.; Arik, L.; Lehto, S. G.; Sun, H.; Hsieh, F.-Y.; Xu, C.; Fremeau, R. T.; Allen, J. R. J. Med. Chem. 2011, 54, 7232. (d) Hirano, J.; Hamase, K.; Zaitsu, K. Tetrahedron 2006, 62, 10065. (e) Ji, X.; Li, D.; Wang, Z.; Tan, M.; Huang, H.; Deng, G. J. Eur. J. Org. Chem. 2017, 6652. (f) Li, M.; Li, L.; Gea, H. Adv. Synth. Catal. 2010, 352, 2445. (g) Markees, D. G.; Schwab, L. S. Helv. Chim. Acta 1972, 55, 1319. (h) Stanislav, R.; Iva, O. Collect. Czech. Chem. Commun. 2014, 69, 822. (i) Taylor, N. J.; Emer, E.; Preshlock, S.; Schedler, M.; Tredwell, M.; Verhoog, S.; Mercier, J.; Genicot, C.; Gouverneur, V. R. J. Am. Chem. Soc. 2017, 139, 8267. (j) Ueno, S.; Shimizu, R.; Maeda, R.; Kuwano, R. Synlett 2012, 23, 1639.

(19) The Merck Index, 13th ed.; Merck \& Co: Whitehouse Station, USA, 2001, p. 618.

(20) (a) Price, J. R. Aust. J. Sci. Res., Ser. A 1949, 2, 272. (b) Maslova, M. M.; Marchenko, N. B.; Polshakov, V. I.; Glushkov, R. G. Khim.Farm. Zh. 1993, 27, 57. (c) Reuman, M.; Eissenstat, M. A.; Weaver, J. D. Tetrahedron Lett. 1994, 35, 8303.

(21) (a) Conrad, M.; Limpach, L. Ber. Dtsch. Chem. Ges. 1887, 20, 944. (b) Conrad, M.; Eckhardt, F. Ber. Dtsch. Chem. Ges. 1889, 22, 73. (c) Meyer, H. Monatsh. Chem. 1906, 27, 255.

(22) (a) Knorr, L.; Fertig, E. Ber. Dtsch. Chem. Ges. 1897, 30, 937. (b) Troeger, J.; Dunker, E. J. Prakt. Chem. 1926, 112, 196. (c) Troeger, J.; Müller, W. Arch. Pharm. (Weinheim, Ger.) 1914, 252, 459.

(23) Frank, J.; Mészáros, Z.; Kömives, T.; Márton, A. F.; Dutka, F. J. Chem. Soc., Perkin Trans. 2 1980, 401.

(24) (a) Dobrowolski, J. C.; Katen, A.; Fraser, B. H.; Bhadbhade, M.; Black, D. StC.; Kumar, N. Tetrahedron Lett. 2016, 57, 5442. (b) Pan, G.-F.; Su, L.; Zhang, Y.-L.; Guo, S.-H.; Wang, Y.-Q. RSC Adv. 2016, 6, 25375.
(25) Derabli, C.; Mahdjoub, S.; Boulcina, R.; Boumoud, B.; Merazig, H.; Debache, A. Chem. Heterocycl. Compd. 2016, 52, 99.

(26) (a) Xia, Y.; Yang, Z.-Y.; Xia, P.; Bastow, K. F.; Tachibana, Y.; Kuo, S.-C.; Hamel, E.; Hackl, T.; Lee, K.-H. J. Med. Chem. 1998, 41, 1155. (b) Xia, Y.; Yang, Z.-Y.; Xia, P.; Bastow, K. F.; Nakanishi, Y.; Lee, K.-H. Bioorg. Med. Chem. Lett. 2000, 10, 699.

(27) (a) Saito, K.; Moriya, Y.; Akiyama, T. Org. Lett. 2015, 17, 3202. (b) Bunce, R. A.; Nammalwar, B. J. Heterocycl. Chem. 2011, 48, 613. (c) Wang, J.-F.; Liao, Y.-X.; Kuo, P.-Y.; Gau, Y.-H.; Yang, D.-Y. Synlett 2006, 2791.

(28) Ding, D.; Li, X.; Wang, X.; Du, Y.; Shen, J. Tetrahedron Lett. 2006, 47, 6997.

(29) Genelot, M.; Dufaud, V.; Djakovitch, L. Tetrahedron 2011, 67, 976.

(30) (a) Coppola, G. M.J. Heterocycl. Chem. 1982, 19, 727. (b) Yoshino, Y.; Kurahashi, T.; Matsubara, S. J. Am. Chem. Soc. 2009, 131, 7494.

(31) Tambe, Y. B.; Somesh, S.; Arunendra, P.; Reddy, L. R. Synth. Commun. 2012, 42, 1341.

(32) Mehra, M. K.; Mukund, P. T.; Arun, V.; Kumar, I.; Dalip, K. Org. Biomol. Chem. 2017, 15, 4956.

(33) Arava, V. R.; Bandatmakuru, S. R. Synthesis 2013, 45, 1039.

(34) Audisio, D.; Messaoudi, S.; Peyrat, J.-F. O.; Brion, J.-D.; Alami, M. J. Org. Chem. 2011, 76, 4995.

(35) Carr, R. M.; Sutherland, D. R. J. Labelled Compd. Radiopharm. 1994, 34, 961.

(36) (a) Almeida, A. I. S.; Silva, A. M. S.; Cavaleiro, J. A. S. Synlett 2010, 462. (b) Cross, R. M.; Monastyrskyi, A.; Mutka, T. S.; Burrows, J. N.; Kyle, D. E.; Manetsch, R. J. Med. Chem. 2010, 53, 7076.

(37) Venkataraman, S.; Barange, D. K.; Pal, M. Tetrahedron Lett. 2006, 47, 7317.

(38) (a) Zhao, T.; Xu, B. Org. Lett. 2010, 12, 212. (b) Hessian, K. O.; Flynn, B. L. Org. Lett. 2005, 8, 243. (c) Cross, R. M.; Manetsch, R. J. Org. Chem. 2010, 75, 8654. 